\title{
A CHARACTERIZATION OF Z-SEPARATING ALGEBRAS
}

\author{
SHANKAR HEGDE
}

\begin{abstract}
Let $A$ be a uniformly closed point separating algebra of bounded real valued functions on a set $X$, containing the constant functions. $A$ is called $z$-separating if whenever $Z_{1}, Z_{2}$ are disjoint zero sets of members of $A$ there is some $f \in A$ with $f\left(Z_{1}\right)=0$ and $f\left(Z_{2}\right)=1$. We prove that $A$ is $z$-separating if and only if $A$ consists of precisely those bounded real valued functions $f$ on $X$ for which $f^{-1}(C)$ is a zero set of some member of $A$ for every closed set $C$ of real line.
\end{abstract}

Introduction. Let $X$ be any set and $A$ be a uniformly closed algebra of bounded real valued functions on $X$ containing the constants and separating the points of $X$. We refer to $A$ as an $S W$-algebra on $X$. Let $\mathscr{Z}[A]$ denote the lattice of zero-sets of members of $A$. The algebra $A$ is called $z$-separating iff whenever $Z_{1}, Z_{2} \in \mathscr{Z}[A]$ with $Z_{1} \cap Z_{2}=\varnothing$ there exists $f \in A$ such that $f\left(Z_{1}\right)=0$ and $f\left(Z_{2}\right)=1$. The $z$-separating algebras were introduced and

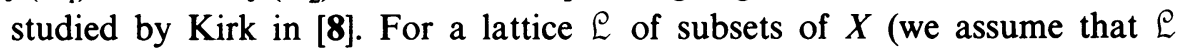

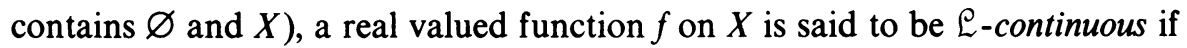
$f^{-1}(C) \in \mathcal{L}$ for every closed set $C$ of $R$, the real line. We denote the algebra of all bounded $\mathcal{L}$-continuous functions on $X$ by $C_{b}(\mathcal{L})$. The purpose of this note is to prove that $A$ is $z$-separating iff $A=C_{b}(\mathscr{Z}[A])$.

For a Tychonoff space $X$, we denote the algebra of all real valued continuous functions on $X$ by $C(X)$ and all the bounded ones by $C_{b}(X)$. Yarious classes of subalgebras of $C(X)$ were studied by Hager [5], [6], [7], Frolik [4] and others. Since an $S W$-algebra $A$ on a set $X$ can always be considered as a subalgebra of $C_{b}(X)$ with a suitable topology on $X$, our study characterizes a class of subalgebras of $C_{b}(X)$. Note that in general a $z$ separating algebra $A$ need not be $C_{b}(X)$ (see Theorem 3.12 and Example 3.5 of Kirk). If $A=C_{b}(X)$, then we always have $A=C_{b}(\mathscr{Z}[A])$. The present note proves that this property characterizes the $z$-separating algebras. More generally, we deduce that $A$ is $z$-separating iff there exists a lattice $\mathcal{L}$ of subsets of $X$ which is closed under countable intersections, such that $A=$ $C_{b}(\mathcal{E})$. In [6] Hager noted that the uniformly closed $c b q$ (closed under bounded quotients) vector lattices $V$ of bounded real valued functions on a set $X$ can be characterized as the families $V$ for which $V=C_{b}(\mathscr{Z}[V])$.

Received by the editors October 31, 1977 and, in revised form, April 6, 1978.

AMS (MOS) subject classifications (1970). Primary 46E25; Secondary 46J10, 46J35.

Key words and phrases. $z$-separating algebra, Wallman compact space, $\mathcal{E}$-continuity and $\mathrm{L}$-uniform continuity. 
Combining this remark with our result it follows that $z$-separating algebras are precisely the $S W$-algebras which are closed under bounded quotients.

1. Preliminaries. Given an $S W$-algebra $A$ on a set $X$, there exists, as a consequence of the Stone-Weierstrass theorem, a compact Hausdorff space $X_{A}$ such that $X$ can be embedded into $X_{A}$ as a dense subspace and $A$ is isomorphic as a Banach lattice to $C_{b}\left(X_{A}\right)$ [2, p. 276]. In fact, $X_{A}$ is the structure space of $A$, that is, the set of all nonzero real homomorphisms of $A$ topologized with the weak star topology. Thus $X_{A}$ is a Hausdorff compactification of $X$ and $A$ consists of precisely those continuous functions on $X$ which can be continuously extended to $X_{A}$. If $Y$ and $Z$ are two compactifications of $X$, we write $Y=Z$ if there exists a homeomorphism of $Y$ onto $Z$ leaving $X$ pointwise fixed.

The idea of the proof of the main theorem is to show that $X_{A}$ is a "Wallman-type compactification" of $X$ and then apply Frink's extension theorem. A lattice $\mathcal{L}$ of subsets of a set $X$ is called strongly normal if it is (i) separating: given $x$ and $y$ in $X, x \neq y$, there exists an $L \in \mathcal{L}$ such that $x \in L$ and $y \notin L$; (ii) disjunctive: for any $L \in \mathcal{L}$ and $x \notin L$, there exists $M \in \mathcal{L}$ such that $x \in M$ and $L \cap M=\varnothing$ and (iii) normal: whenever $L, M \in \mathcal{C}$ with $L \cap M=\varnothing$, there exists $U, V \in \mathcal{L}$ such that $L \subset X-U, M \subset X-V$ and $U \cup V=X$. Given a strongly normal lattice $\mathcal{L}$ of subsets of $X$, we topologize the set $\omega(\mathscr{L})$ of all $\mathcal{L}$-ultrafilters by taking a base for the closed sets, the sets of the form $\bar{L}=\{\mathscr{Q} \in \omega(\mathcal{L}): L \in \mathcal{Q}\}$ where $L \in \mathcal{L}$. Then $\omega(\mathcal{L})$ is a compact Hausdorff space and the mapping $x \rightarrow \mathscr{U}_{x}$ where $\mathscr{U}_{x}$ is the $\mathcal{L}$-ultrafilter consisting of all $L \in \mathcal{L}$ for which $x \in L$, is an embedding of $X$ into $\omega(\mathcal{E})$ and $X$, treated as a subspace of $\omega(\mathcal{L})$ is dense in it (see Brooks [1]). We refer to $\omega(\mathcal{L})$ as the Wallman compact space relative to $\mathcal{L}$. Given a lattice $\mathcal{E}$ of subsets of $X$, a real valued function $f$ on $X$ is said to be $\mathcal{L}$-uniformly continuous if for each $\varepsilon>0$, there exists a finite family $\left\{L_{1}, \ldots, L_{n}\right\}$ of members of $\mathcal{E}$ such that $\bigcap_{i=1}^{n} L_{i}=\varnothing$ and the oscillation of $f$ on each of the sets $X-L_{i}$ is less than $\varepsilon$. The following extension theorem is proved in [3].

THEOREM 1.1 (O. FRINK). Let $\mathcal{L}$ be a strongly normal lattice of subsets of $X$. For each real valued function $f$ on $X$, there exists an extension $\bar{f}$ of $f$ to $\omega(\mathcal{L})$ such that $\bar{f} \in C(\omega(\mathcal{L}))$ iff $f$ is $\mathcal{L}$-uniformly continuous.

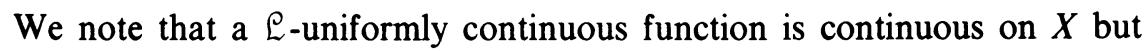
need not be $\mathcal{L}$-continuous on $X$.

2. The results. In this section we prove the theorem stated in the introduction and deduce some interesting corollaries. First we prove a few lemmas.

LeMma 2.1. Let $A$ be an $S W$-algebra on a set $X$. Then (i) $\mathscr{Z}[A]$ is a strongly normal lattice on $X$. (ii) $X_{A}=\omega(\mathscr{Z}[A])$ iff $A$ is $z$-separating.

Proof. Equip $X$ with the relative topology inherited from the topology of 
$X_{A}$. Since $\mathscr{Z}[A]$ is the trace of zero sets of $X_{A}$ on $X$, the results follow from Theorems 5.3 (b) and 5.2 of Hager [5].

REMARKS. If $T$ is a compactification of a Tychonoff space $X$ and $\mathscr{Z}_{T}$ denotes the trace of the zero-sets of $T$ on $X$, then Hager [5] has proved that $\mathscr{Z}_{T}$ is a normal base on $X$ and $\omega\left(\mathscr{Z}_{T}\right)$ coincides with the structure space of $A(T)$, where $A(T)$ is the smallest uniformly closed, inverse closed subalgebra of $C(X)$ containing all those members which are continuously extendible to $T$. Then by Lemma 2.1 it follows that $A$ is $z$-separating iff $X_{A}$ is the structure space of the inverse closed algebra $A\left(X_{A}\right)$. Thus in this case $A$ consists of precisely the bounded members of $A\left(X_{A}\right)$.

LEMMA 2.2 For any lattice $\mathcal{L}$ of subsets of a set $X$, every bounded $\mathcal{L}$ continuous function on $X$ is $\mathcal{L}$-uniformly continuous.

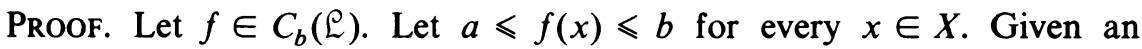
arbitrary $\varepsilon>0$, let $0<\delta<\varepsilon \mid 2$. Choose $a_{1}, a_{2}, \ldots, a_{n}$ in $[a, b]$ such that $[a, b] \subset \cup_{i=1}^{n}\left(a_{i}-\delta, a_{i}+\delta\right)$. Then for $i=1, \ldots, n, L_{i}=f^{-1}\left(-\infty, a_{i}-\delta\right]$ $\cup f^{-1}\left[a_{i}+\delta, \infty\right) \in \mathcal{L}$. Now it is readily verified that $\cap_{i=1}^{n} L_{i}=\varnothing$ and the oscillation of $f$ on each of the sets $X-L_{i}$ is less than $\varepsilon$. Thus $f \in C_{u}(\mathcal{E})$, where $C_{u}(\mathfrak{L})$ denotes the set of all $\mathcal{L}$-uniformly continuous functions on $X$.

A family of sets is called a delta family if it is closed under countable intersections. Next we prove

LEMMA 2.3. If $\mathcal{L}$ is a strongly normal delta lattice of subsets of a set $X$, then $C_{b}(\mathcal{L})=C_{u}(\mathcal{E})$.

Proof. In view of Lemma 2.2, it is enough to show that $C_{u}(\mathfrak{L}) \subset C_{b}(\mathfrak{E})$. First we note that for a delta lattice $\mathcal{L}, f \in C_{b}(\mathcal{L})$ iff $f^{-1}(-\infty, a]$ and $f^{-1}[b, \infty) \in \mathcal{L}$ for all real numbers $a$ and $b$.

Recall the construction of $\omega(\mathcal{L})$. The family $\overline{\mathcal{L}}=\{\bar{L}: L \in \mathcal{L}\}$ where $\bar{L}$ consists of $\mathcal{L}$-ultrafilters of which $L$ is a member, is a base for the closed sets of $\omega(\mathcal{L})$. Also it is easy to see that $\overline{\mathcal{L}}$ is a strongly normal lattice of subsets of $\omega(\mathcal{L})$. Let $\rho(\overline{\mathcal{E}})$ denote the delta lattice consisting of countable intersections of members of $\overline{\mathrm{E}}$. One can check that $C_{b}(\rho(\overline{\mathcal{E}}))$ is an $S W$-algebra on the compact Hausdorff space $\omega(\mathcal{L})$ and hence by the Stone-Weierstrass theorem, $C_{b}(\rho(\overline{\mathcal{E}}))$ $=C(\omega(\mathfrak{L}))$.

Let $f \in C_{u}(\mathfrak{L})$. Let $\bar{f}$ denote the unique continuous extension of $f$ to $\omega(\mathfrak{L})$. Let $a \in R$. Then $\bar{f}^{-1}(-\infty, a]=\cap_{n=1}^{\infty} \bar{B}_{n}$ for some sequence $\left\{B_{n}\right\}$ in $\mathcal{L}$. Hence $f^{-1}(-\infty, a]=X \cap \bar{f}^{-1}(-\infty, a]=\cap_{n=1}^{\infty}\left(X \cap \bar{B}_{n}\right)=\cap_{n=1}^{\infty} B_{n}$ since each $B_{n} \in \mathcal{L}$ is closed in $X$. Now $\mathcal{L}$ being a delta lattice, $f^{-1}(-\infty, a] \in \mathcal{L}$. Similarly it follows that $f^{-1}[b, \infty) \in \mathcal{L}$ for every $b \in R$. Thus $f \in C_{b}(\mathcal{L})$. This completes the proof.

In particular, given any $S W$-algebra $A$ on a set $X$ we always have $C_{b}(\mathscr{Z}[A])$ $=C_{u}(\mathscr{Z}[A])$. 
THEOREM 2.4. Let $A$ be an $S W$-algebra on a set $X$. Then $A$ is $z$-separating iff $A=C_{b}(\mathscr{Z}[A])$.

Proof. Suppose $A$ is $z$-separating. Let $f \in C_{b}(\mathscr{Z}[A])$. By Lemma 2.3 and Theorem 1.1, $f$ can be extended to be a continuous function on $\omega(\mathscr{Z}[A])$. By Lemma $2.1 X_{A}=\omega(\mathscr{Z}[A])$ so that there is an isomorphism of $C(\omega(\mathscr{Z}[A]))$ onto $C\left(X_{A}\right)$ such that the corresponding elements under this isomorphism agree on $X$. Hence $f$ has a continuous extension $\bar{f} \in C\left(X_{A}\right)$. Since $A$ consists of precisely the restrictions of members of $C\left(X_{A}\right)$ to $X, f \in A$. Thus $C_{b}(\mathscr{Z}[A])$ $\subset A$.

On the other hand we note that for any $S W$-algebra $A$ we have $A \subset$ $C_{b}(\mathscr{Z}[A])$. In fact, since $A$ contains the constants and is closed under the lattice operations, for every pair of real numbers $a$ and $b$ and every $f \in A$, $g=\max (f, a)-a \in A$ and $h=b-\min (b, f) \in A$. Hence $f^{-1}(-\infty, a]=$ $Z(g) \in \mathscr{Z}[A], f^{-1}[b, \infty)=Z(h) \in \mathscr{Z}[A]$. This proves that $A=C_{b}(\mathscr{Z}[A])$.

Conversely suppose $A=C_{b}(\mathscr{Z}[A])$. As is well known $C_{b}(\mathscr{Z}[A])$ is closed under bounded quotients (see e.g. [7]). Let $Z_{1}=Z(f), Z_{2}=Z(g)$ be disjoint members of $\mathscr{Z}[\mathrm{A}]$ where $f, g \in A$. Let $h=|f|(|f|+|g|)^{-1}$. Then $0 \leqslant h \leqslant 1$. Hence $h \in A$. Furthermore $h\left(Z_{1}\right)=0$ and $h\left(Z_{2}\right)=1$, proving that $A$ is $z$-separating.

As immediate consequences we have

COROLlaRY 2.5. For any $S W$-algebra $A$ on a set $X, C_{b}(\mathscr{Z}[A])$ is the smallest $z$-separating algebra containing $A$.

COROLlaRY 2.6 (KIRK). Let $X$ be a Tychonoff space and $A$ be a $z$-separating subalgebra of $C_{b}(X)$. Then $A=C_{b}(X)$ iff $\mathscr{Z}[A]=\mathscr{Z}\left[C_{b}(X)\right]$.

We further deduce

Corollary 2.7. Let $A$ be an $S W$-algebra on a set $X$. Then $A$ is $z$-separating iff there exists a delta lattice $\mathcal{L}$ of subsets of $X$ such that $A=C_{b}(\mathcal{E})$.

Proof. For a delta lattice $\mathcal{L}$ of subsets of $X$, one can readily check that

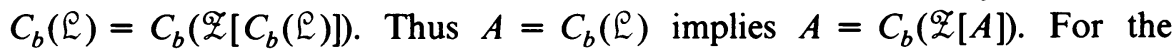
converse take $\mathcal{L}=\mathscr{Z}[A]$.

REMARKS. In [1] Brooks pointed out that if $\mathcal{L}$ is a strongly normal lattice of subsets of $X$, then the finite covers of $X$ from the family of $\mathcal{L}$-complements generate a separated uniform structure on $X$ and $C_{u}(\mathfrak{L})$ is precisely the family of bounded real valued functions on $X$ which are uniformly continuous relative to this structure. In the light of this remark compare our results with those of Frolik [4] on uniform spaces.

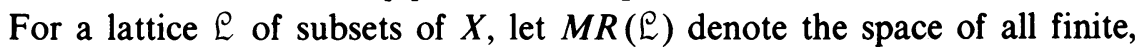

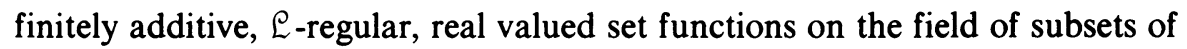
$X$ generated by $\mathcal{L} . M R(\mathscr{L})$ is said to represent $A^{*}$, the Banach dual of $A$, if there exists a (unique) Banach lattice isomorphism $I$ from $A^{*}$ onto $M R(\mathcal{L})$ 
such that for each nonnegative $\psi \in A^{*}, I \psi(W)=\inf \left\{\psi(f): f \in A, \mathfrak{X}_{W} \leqslant\right.$ $f\}$ for every $W \in \mathcal{L}$. Kirk [8] has proved that $M R(\mathscr{Z}[A])$ represents $A^{*}$ iff $A$ is $z$-separating. Thus we obtain

Corollary 2.8. $M R(\mathscr{Z}[A])$ represents $A^{*}$ iff $A=C_{b}(\mathscr{Z}[A])$.

The author is grateful to Professor P. Cuttle for helpful discussions and wishes to thank the referee for his comments concerning the works of Hager and Frolik.

\section{REFERENCES}

1. R. M. Brooks, On Wallman compactifications, Fund. Math. 60 (1967), 157-173.

2. N. Dunford and J. T. Schwartz, Linear operators Part I, Interscience, New York, 1958.

3. O. Frink, Compactifications and seminormal spaces, Amer. J. Math. 86 (1964), 602-607.

4. Z. Frolik, Three uniformities associated with uniformly continuous functions, Symposia Math. 17 (1976), 69-80.

5. A. W. Hager, On inverse closed subalgebras of $C(X)$, Proc. London Math. Soc. 19 (1969), 233-257.

6. _ Real-valued functions on Alexandroff (zero-set) spaces, Comment. Math. Univ. Carolinae 16 (1975), 755-769.

7.,$A$ class of function algebras (and compactifications and uniform spaces), Symposia Math. 17 (1976), 11-23.

8. R. B. Kirk, Algebras of bounded real valued functions. I, II, Nederl. Akad. Wetensh. Proc. Ser. A 75 = Indag. Math. 34 (1972), 443-463.

Department of Mathematics, University of Saskatchewan, Saskatoon, 'Saskatchewan, CanADA S7N OWO

Current address: Department of Mathematical Sciences, Northern Illinois University, DeKalb, Illinois 60115 